\title{
Anatomical variations of the coeliac trunk in the homogeneous Polish population
}

\author{
K. Torres ${ }^{1}$, G. Staśkiewicz ${ }^{1}, 2$, M. Denisow ${ }^{1}$, Ł. Pietrzyk ${ }^{1}$, A. Torres ${ }^{1}$, M. Szukała1, \\ E. Czekajska-Chehab², A. Drop² \\ ${ }^{1}$ Human Anatomy Department, Medical University of Lublin, Poland \\ ${ }^{2}$ Department of Radiology and Nuclear Medicine, Medical University of Lublin, Poland
}

[Received 19 June 2014; Accepted 10 August 2014]

Background: The abdominal vessels show a number of abnormalities and pathologies knowledge of which is important during open as well as laparoscopic surgeries. One of the most common vessels which pattern has many variations is the coeliac trunk. The present study was undertaken on homogeneous population to assess morphology of the coeliac trunk and to evaluate the possible variability of its branches.

Materials and methods: Standard abdominal multidetector computed tomography examinations were performed on a total of 1569 patients diagnosed by 2 radiologists independently; in case of doubts common assessment was performed. Results: The coeliac trunk followed the classic pattern in $92.7 \%$ of the cases (1455/1569 of patients). The gastrosplenic trunk was detected in $4.1 \%$ of cases (64/1569); the hepatosplenic trunk in 2.2\% of cases (34/1569); the coeliac-mesenteric trunk was observed in $0.5 \%$ of cases (8/1569); the hepatogastric trunk in $0.2 \%$ of cases (4/1569); the splenomesenteric trunk was detected in $0.1 \%$ of cases (2/1569). In the next $0.1 \%$ the coeliac trunk was absent. The hepatosplenomesenteric and the coeliac-colic trunk were not observed in the study population. Conclusions: Vascular anatomical abnormality is usually asymptomatic hovewer, its preoperative knowledge is helpful to reduce complications like vascular bleeding when dissecting the hapato-pancreatic region. The coeliac trunk and its branches can be fast and easy evaluated in computed tomography exams performed due to various symptoms from abdominal cavity. (Folia Morphol 2015; 74, 1: 93-99)

Key words: anatomic variations, coeliac trunk, mulitdetector computed tomographic, angiography, gastrosplenic trunk, coeliac-colic trunk

\section{INTRODUCTION}

The coeliac trunk is first branch of the abdominal aorta arising at the level of Th12-L1 vertebrae. The coeliac trunk trifurcates into the left gastric, common hepatic and splenic arteries [37]. The trifurcation of the coeliac trunk was first described by Haller in 1756 and is still considered to be the classical appearance of the coeliac trunk [20]. Sometimes the coeliac trunk bifurcates into the splenic and the hepatic artery; in this case the origin of the left gastric artery is mobile and can slide on the aorta, all over the coeliac trunk up to a trifurcation [36].

The abdominal vessels show a number of abnormalities and pathologies. One of the most common vessel which pattern has many variations is the coeliac

Address for correspondence: K. Torres, MD, PhD, MBA, Human Anatomy Department, Medical University of Lublin, ul. Jaczewskiego 4, 20-094 Lublin, Poland, fax: +48 8152888 18, e-mail: kamiltorres@wp.pl 
trunk [35]. In the most cases the coeliac trunk follows the classic patter while gastrosplenic and hepatosplenic trunk are most common variations described in the literature $[10,15]$.

Aneurysms of the abdominal aorta branches are most frequently found during laboratory investigations performed for various clinical purposes [27]. Raptures of the splanchnic aneurysms occur in 20-22\% of patients and result in enormous hemorrhage with mortality rate ranging from $35 \%$ to $100 \%[4,9,26,31]$.

Vascular abnormalities can lead to various complications, e.g. blood loss during endovascular procedures in the abdominal cavity $[13,14]$.

Knowledge of coeliac trunk variations is of great value during surgical interventions involving duodenum and pancreas and radiological management $[20,22]$. It is also essential for liver transplantation and resection, hepatic artery infusion chemotherapy, gastrectomy, or biliary reconstruction [22]. Vascular variations of the abdominal aorta and its branches have practical significance during kidney transplantation and oncological resections [23].

Non-invasive identification of the coeliac trunk and its branches is possible during multidetector computed tomography (MDCT) studies [35].

The purpose of the study was to assess morphology of the coeliac trunk and variability of its branches in homogeneous population using MDCT.

\section{MATERIALS AND METHODS}

All patients who underwent abdominal CT studies in the Department of Radiology, Clinical Hospital No. 4 in Lublin, Poland in 2012 were investigated retrospectively. The study population consists of 1569 patients ( 829 females and 740 males). The mean age of patients was 58.0 (age range: 16-96) years.

Abdominal CT studies were performed using a 64-row scanner (Lightspeed CT, GE Medical Systems). During examination approximately $80 \mathrm{~mL}$ of non-ionic iodinated contrast was injected into patients' antecubital vein. Scanning was performed with standard protocol used at our institution: collimation, $2.5 \mathrm{~mm}$; gantry rotation time, $0.5 \mathrm{~s} ; \mathrm{mAs}$ modulation, $120 \mathrm{kV}$. 50-90 $\mathrm{mL}$ (depending on patient body weight) of non-ionic contrast medium followed $30 \mathrm{~mL} 0.9 \mathrm{NaCl}$ was injected at $3.0 \mathrm{~mL} / \mathrm{s}$ through an antecubital vein with an automatic dual power injector. Images were obtained from the level of the diaphragm to the iliac wings. CT studies were reviewed on at the workstation Advantage Window 4.6 with multiplanar
Table 1. Uflacker's classification used in the study

\begin{tabular}{ll}
\hline Type & Variation \\
\hline I & Classic coeliac trunk \\
II & Hepatosplenic trunk \\
III & Hepatogastric trunk \\
IV & Hepatosplenomesenteric trunk \\
V & Gastrosplenic trunk \\
VI & Coeliac-mesenteric trunk \\
VII & Coeliac-colic trunk \\
VIII & No coeliac trunk \\
\hline
\end{tabular}

Table 2. Coeliac trunk variations according to Uflacker's classification found in the study

\begin{tabular}{llc}
\hline Type & & No. (\%) of cases \\
\hline I & Classic coeliac trunk & $1455(92.7 \%)$ \\
II & Hepatosplenic trunk & $34(2.2 \%)$ \\
III & Hepatogastric trunk & $4(0.2 \%)$ \\
IV & Hepatosplenomesenteric trunk & $0(0 \%)$ \\
V & Gastrosplenic trunk & $64(4.1 \%)$ \\
VI & Coeliac-mesenteric trunk & $8(0.5 \%)$ \\
VII & Coeliac-colic trunk & $0(0 \%)$ \\
VIII & No coeliac trunk & $2(0.1 \%)$ \\
\hline
\end{tabular}

reformations (MPR), maximum intensity projections (MIP) and volume rendering technique (VR).

The pattern of the aortic origin of the four major arteries: left gastric, the common hepatic, splenic and superior mesenteric arteries were analysed in the study. Two radiologists performed the evaluation of abdominal CT studies independently and in case of doubts common assessment was performed. Identification of coeliac trunk and its branches was possible in all patients examined. The instructional 3-dimensional (3D) models of the coeliac trunk and its abnormalities were designed.

Anatomical variations of the coeliac trunk were described according to Uflacker's classification (Table 1) [34].

\section{RESULTS}

In line with Uflacker's classification schema (Table 1), the normal anatomy (type I) of the coeliac trunk dominated in the studied homogeneous Polish population and was recorded in 1455 of the 1569 patients (92.7\%) (Table 2). Classic coeliac trunk is the artery branching form the abdominal aorta and trifurcates into the left gastric, splenic and common hepatic artery (Fig. 1). 


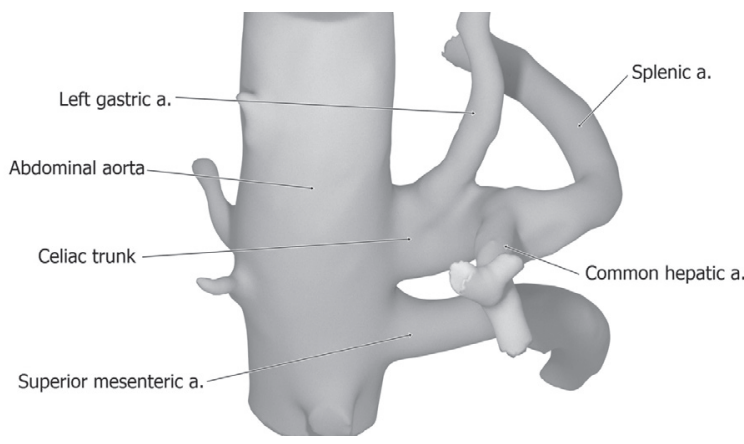

Figure 1. Three-dimensional model of the classic coeliac trunk.

The gastrosplenic trunk (type V) was the most prevalent variation ( $4.1 \%$ of cases) followed by hepatosplenic trunk (type II) (2.2\% of cases). Gastrosplenic trunk is recognised as the common origin of the left gastric and splenic artery (Fig. 2). Common origin of the common hepatic and splenic artery is classified as the hepatosplenic trunk. In this case the left gastric artery arises directly from the abdominal aorta (Fig. 3).
The other coeliac trunk abnomalities were very rare - the coeliac-mesenteric trunk (type VI) was observed in $0.5 \%$ of cases; the hepatogastric trunk (type III) in $0.2 \%$ of cases. Common origin of two unpaired visceral branches of the abdominal aorta: the coeliac trunk and the superior mesenteric artery (SMA) form the coeliac-mesenteric trunk (Fig. 4). Hepatogastric trunk is formed by common origin of the common hepatic and left gastric artery. When this variation is present the splenic artery is a direct branch of the abdominal aorta (Fig. 5).

In the next $0.1 \%$ of cases the coeliac trunk was absent (type VIII). No coeliac trunk is recognised while left gastric, common hepatic and splenic arteries arise directly from the abdominal aorta (Fig. 6).

The hepatosplenomesenteric (type IV) and the coeliac-colic trunk (type VII) were not observed in the study population. Common origin of 3 abdominal arteries such as the common hepatic artery, splenic and SMA is defined as the hepatosplenomesenteric trunk. In this case the left gastric artery branches directly from the abdominal aorta. The coeliac-colic
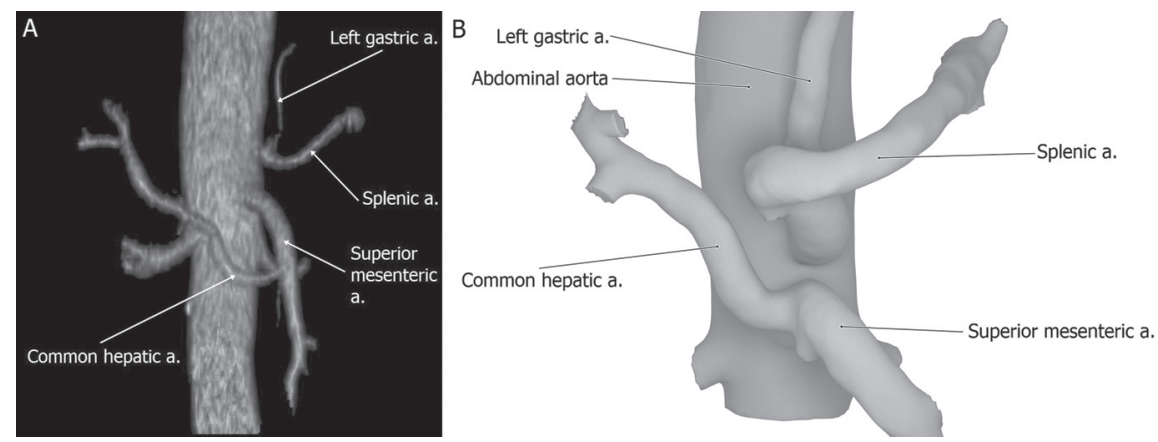

Figure 2. Reconstruction of the gastrosplenic trunk; A. Right oblique multidetector computed tomography volume rendering reconstruction; B. Three-dimensional model.
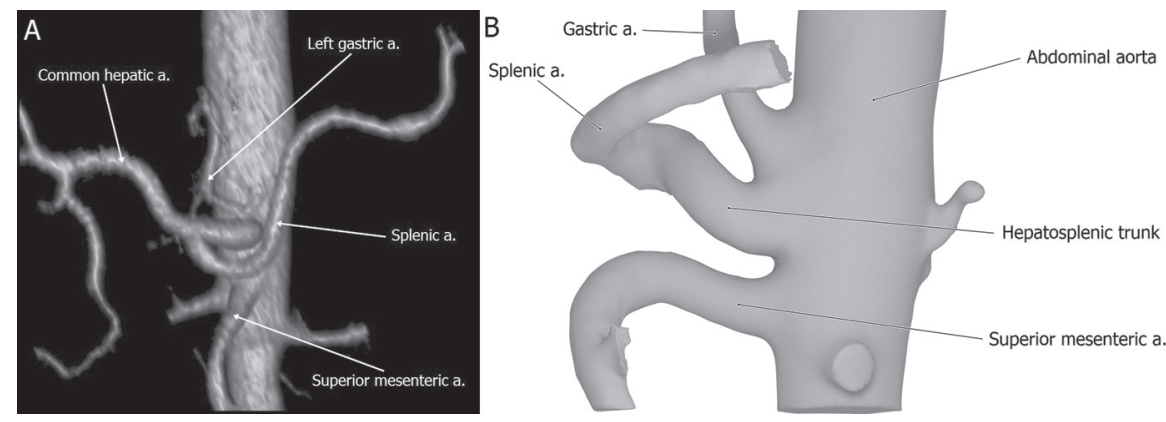

Figure 3. Reconstruction of the hepatosplenic trunk; A. Anterior multidetector computed tomography volume rendering reconstruction; B. Three-dimensional model. 

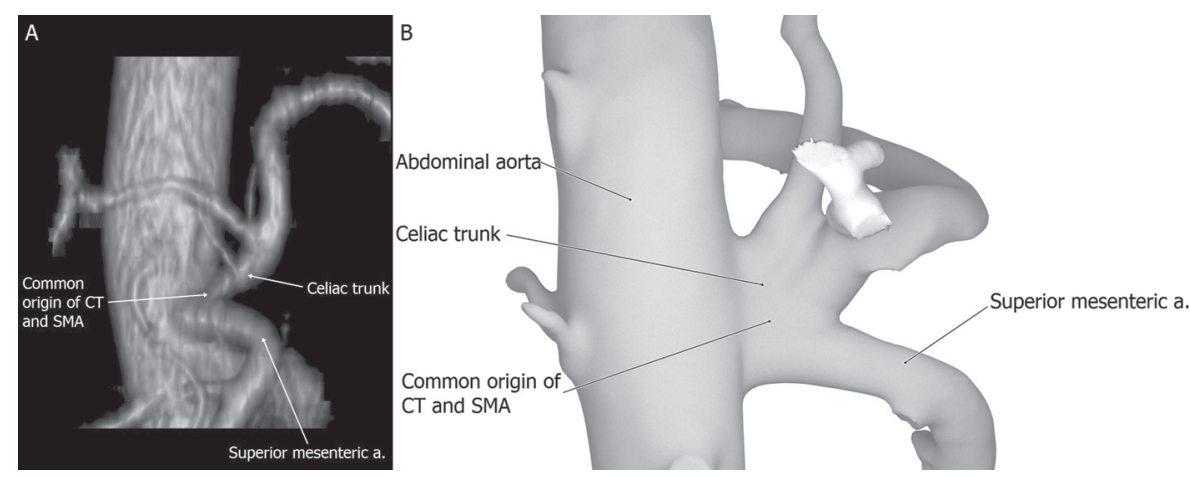

Figure 4. Reconstruction of the coeliac-mesenteric trunk; A. Right oblique multidetector CT volume rendering reconstruction; B. Three-dimensional model; CT — computed tomography; SMA — superior mesenteric artery.
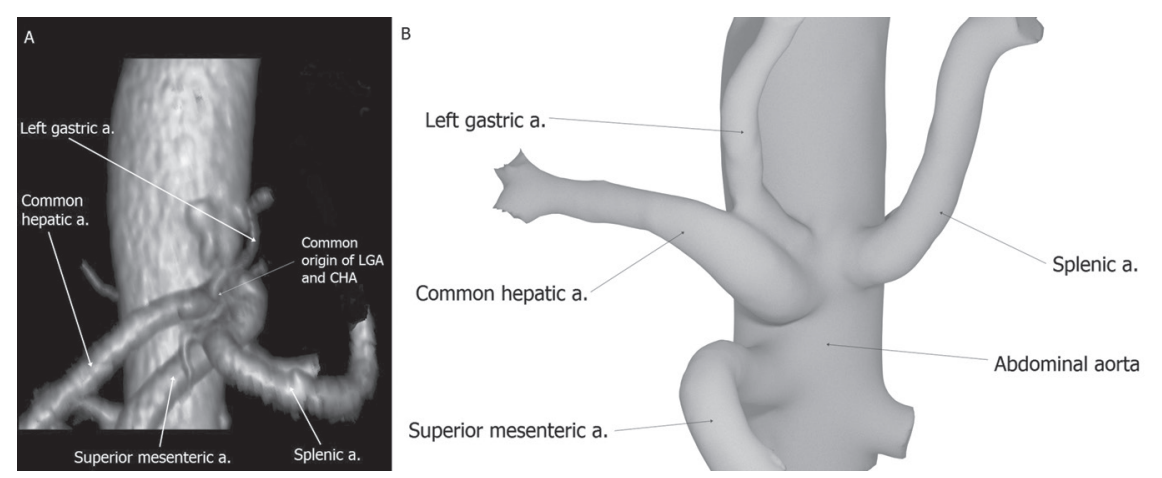

Figure 5. Reconstruction of the hepatogastric trunk; A. Right oblique multidetector computed tomography volume rendering reconstruction; B. Three-dimensional model; CHA — common hepatic artery; LGA — left gastric artery.
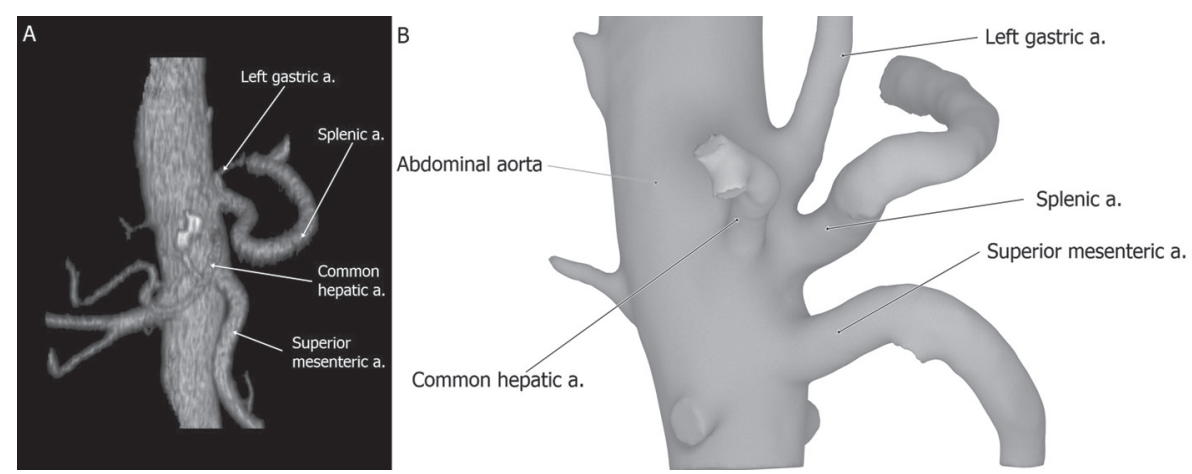

Figure 6. Reconstruction of the absent coeliac trunk; A. Right oblique multidetector computed tomography volume rendering; B. Three-dimensional model.
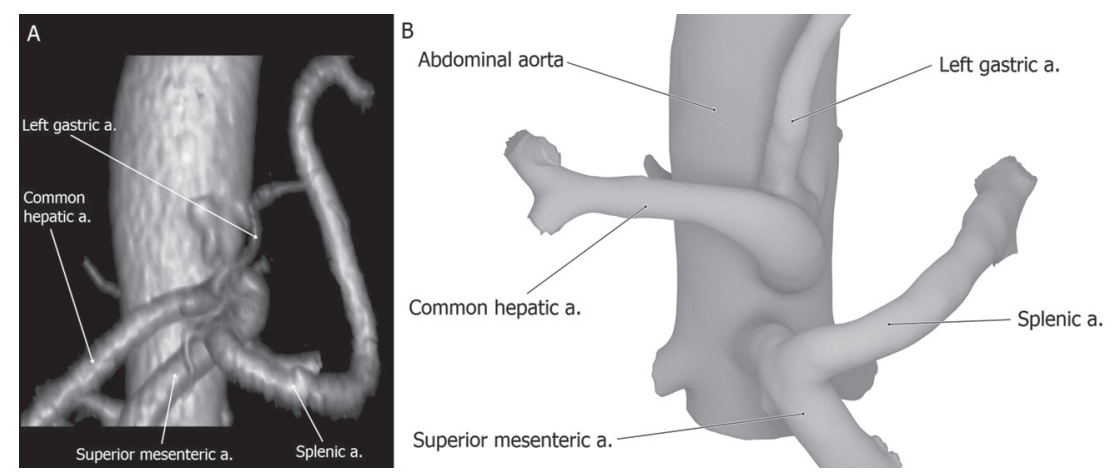

Figure 7. Reconstruction of the splenomesenteric trunk; A. Right oblique multidetector computed tomography volume rendering; B. Three-dimensional model. 
trunk is formed when the middle colic artery originates from the coeliac trunk instead of the SMA.

Additionally, there were $2(0.1 \%)$ cases of splenomesenteric trunk, which was not decribed in the Uflacker's classification. Splenomesenteric trunk is formed by the common origin of the splenic and SMA (Fig. 7). Usually splenomesenteric trunk is accompanied by the common origin of the common hepatic and left gastric artery forming the hepatogastric trunk.

\section{DISCUSSION}

\section{Embryology}

Anatomical variations of the coeliac trunk and other branches of the abdominal aorta results from foetal developmental changes in the ventral segmental arteries supplying the digestive tube [16]. The roots $10^{\text {th }}-13^{\text {th }}$ give origin for the left gastric artery, common hepatic artery, splenic artery, and SMA, respectively. According to Tandler, these ventral segmental arteries are connected with the longitudinal anastomosis running parallel to the abdominal aorta, forming dorsal and ventral segmental (splanchnic) anastomosis [33]. In further development first, second and third roots coalesce to form coeliac trunk whereas single fourth root gives origin for the SMA and they still remain connected through the ventral longitudinal anastomosis. Separation of the ventral longitudinal anastomosis occurring at unusual level results in displacement of origin of coeliac trunk branches and SMA [14, 25, 29].

\section{Classic coeliac trunk}

In the literature, classic coeliac trunk, type in I in Uflacker's classification, has been reported with a frequency of $72.0 \%$ or $89.0 \%$ [15, 35, 37]. The occurance of classical coeliac trunk trifurcation found in our study (92.7\% of cases) concurs with Gümüs et al. [10] who examined 820 patients and documented type I in $91.7 \%$ of cases. Despite the fact that isolated aneurysms of the coeliac trunk are very rare, $4 \%$ of all visceral arteries aneurysms, they can be easy detected and its classic pattern reduce a number of complications during surgical approaches or endovascular embolisation [2].

\section{Hepatosplenic trunk}

Hepatosplenic trunk, type II in the Uflacker's classification, was described by Song et al. [30] as the most common coeliac trunk variation with frequency of $4.4 \%$ cases. In our study, type II abnomality was observed as the second common variation, concurently to Gümüs et. al. [10], however different frequency was noted (3\% and $2.2 \%$, respectively). lezzi et al. [12] performing MDCT angiographs diagnosed the hepatosplenic trunk in $2.7 \%$ of cases (14/524).

\section{Hepatogastric trunk}

We found hepatogastric trunk, type III in the Uflacker's classification, in 4 of 1569 (0.2\%) cases. Variation type III is described with different frequency. Ugurel et al. [35] found it in 1\% of cases. In turn no hepatogastric trunk was observed in the study of Gümüs et al. [10]. This anomaly is usually found incidentally however $5 \%$ (26 of 524) of the common origin of the common hepatic artery and left gastric artery was reported by Yíldírím et al. [38].

\section{Hepatosplenomesenteric trunk}

No case of hepatosplenomesenteric trunk (type IV) was reported in our study. This variation was reported by Chen et al. [7] in $0.7 \%$ of cases and is close the frequency noted by Ugurel et al. [35] who described 1 case of type IV variation (1\%). Occurrence at the level of $0.5 \%$ was described by Bergman et al. [5]. This pathology seems to be crucial when performing pancreatic resection. Then the blood supply to the duodenum may come only from the SMA. Accidental ligation of the SMA or branches of the common trunk may lead to the ischaemia or even necrosis of the liver or duodenum [3, 29].

\section{Gastrosplenic trunk}

In our study, the gastrosplenic trunk, the type V, was present in $4.1 \%$ of cases (64 of 1569 patients) and was the most common abnormality of the coeliac trunk. It is in accordance with Ugurel et al. [35], who documented gastrosplenic trunk in $4 \%$ of 100 Turkish patients, and also recognised the variation as most common. Gümüs et al [10] observed gastrosplenic trunk with the frequency of $2.6 \%$ (21 of 820 cases). The incidence of gastrosplenic trunk at the level of $1.3 \%$ was observed by Venieratos et al. [37] who dissected 77 adult cadavers of Caucasian (Hellenic) origin. The occurrence of the gastrosplenic trunk was variable in Japanese population. Adachi et al. [1] found only 1 case out of 252 specimens (0.4\%), while Nakamura et al. [19] documented $1.1 \%$ of cases during gross-anatomy dissection of 275 cadavers. It seems that the origin of population impact on the type $\mathrm{V}$ anatomical anomaly of coeliac trunk but the factors other than the origin cannot be excluded. 


\section{Coeliac-mesenteric trunk}

In our study the type VI - coeliac-mesenteric trunk was present in 8 of 1569 cases $(0.5 \%)$. The common origin of the coeliac trunk and the SMA is known by arc of Bühler's [17] and was identified in $3.3 \%$ by Saad et al. [24] and in 14 cases of 340 arteriograms (4.1\%) by Grabbe et al. [8]. However, there is also a report of no coeliac-mesenteric trunk when examining 100 cases [35]. Preoperative knowledge of this anomaly is extremely important when planning pancreaticoduodenectomy which is the option for pancreatic and peripancreatic cancers treatment. The incidence of coeliac-mesenteric trunk increases a perioperative morbidity by $20-30 \%$ [28]. However, the proper coeliac-mesenteric trunk identification may help in choosing better treatment option and may both avoid iatrogenic injury and decrease the incidence of death.

\section{Coeliac-colic trunk}

Type VII - coeliac-colic trunk is formed when the middle colic artery originates from the coeliac trunk instead of the SMA. Coeliac-colic trunk, described by Uflacker is a sporadic anatomical aberrance described with a rate of $0.5-1 \%$ in cadaver studies, and was not revealed by Ugurel et al. [35] as well as was absent in our examinations $[21,34]$.

Despite low frequency it may lead to difficulties and complications during transverse colon surgery. In most cases the coeliac-colic trunk receives blood form SMA and inferior mesenteric artery, which may result in unexpected bleeding during surgical procedures.

\section{No coeliac trunk}

Type VIII in the Uflacker's classification - no coeliac trunk is recognised while left gastric, common hepatic and splenic arteries arise directly from the abdominal aorta. Absence of CT, was identified as a morphological type firstly in the Morita's classification [18]. In our study absent of CT was found in $0.1 \%$ of cases and corresponds an earlier reports of Nelson et al. [21] - 0.19\%. Slightly higher level ( $0.35 \%$ of cases) was documented by Hiatt et al. [11]. The highest frequency of the type VIII variation (4.0\%) was described by Prakash et al. [23] in cadaveric study.

\section{Splenomesenteric trunk}

In our study splenomesenteric trunk, not classified by Uflacker, was present in $0.1 \%$ of cases. Splenome- senteric trunk is described in less than $1 \%[26,32]$. Ugurel et al. [35] have reported it in 1\% of cases and Suma et al. [32] detected it in 1.2\%. Bergman et al. [6] reported the origin of the superior mesenteric and splenic artery as a common trunk in $0.5 \%$ of population.

Aneurysm involving splenic artery accounts approximately $6 \%$ of all visceral aneurysms while SMA is involved in the pathological process in $5.5 \%$ of cases [9]. Aneurysms of the common trunk of splenic and mesenteric arteries are very rare. Settembrini et al. [27] described 2 cases of 4-cm aneurysms of the splenomesenteric trunk in young patients. Preoperative plan of the surgical treatment of aneurysms should take into account theirs size and anatomical conditions. It is especially important to be aware of the presence of splenomesenteric variations what makes the surgical approach difficult due to complicated anatomical location and close relation to the pancreas, portal vein and common bile duct.

\section{CONCLUSIONS}

Vascular variations are usually asymptomatic; however, its preoperative knowledge is helpful to reduce any potential risks like vascular bleeding when dissecting in the hapato-pancreatic region, an integral part of gastrointestinal surgeries. The evaluation of coeliac trunk and its branches is possible in CT examination performed due to symptoms from abdominal cavity.

\section{REFERENCES}

1. Adachi B (1928) Das Arteriensystem der Japaner, Band II. Verlag der Kaiserlich-Japanischen Universität zu Kyoto, p. 28, 38, 54 .

2. Al-Wahbi AM (2011) Giant celiac artery aneurysm: treatment by transcatheter coil embolization. Int J Surg Case Rep, 2: 191-193.

3. Anson BJ, McVay CB (1984) Surgical anatomy. 6th Ed. Saunders, Philadelphia, p. 692.

4. Bailey RW, Riles TS, Rosen RJ, Sullivan LP (1991) Celiomesenteric anomaly and aneurysrn: clinical and etiologic features. J Vasc Surg, 14: 229-234.

5. Bergman RA, Afifi AK, Miyauchi R (2007) Celiak trunk arteries. Illustrated encyclopedia of human anatomic variation. Opus II: cardiovascular system: arteries: abdomen, http://www.Anatomyatlases.org/AnatomicVariants/ Cardiovascular/Text/Arteries/CeliacTrunk.shtml [accessed February 2014].

6. Bergman RA, Afifi AK, Saadch FA (1988) Compendium of human anatomic variation: Text, atlas, and world literature. Urban-Schwarzenberg, Munich, p. 163.

7. Chen H, Yano R, Emura S, Shoumura S (2009) Anatomic variation of the celiac trunk with special reference to hepatic artery patterns. Ann Anat, 191: 399-407. 
8. Grabbe E, Bücheler E (1980) Die Bühlersche Anastomose. Bühler's Anastomosis Stuttgart. Georg Thieme Verlag, New York 132: 541-546.

9. Graham LM, Stanley JC, Whitehouse WM Jr, Zelenock GB, Wakefield TW, Cronenwett JL, Lindenauer SM (1985) Celiac artery aneurysms: historic (1745-1949) versus contemporary (1950-1984) differences in etiology and clinical importance. J Vasc Surg, 2: 757-764.

10. Gümüs H, Bükte Y, Ozdemir E, Sentürk S, Tekbas G, Onder H, Ekici F, Bilici A (2013) Variations of the celiac trunk and hepatic arteries: a study with 64-detector computed tomographic angiography. Eur Rev Med Pharmacol Sci, 17: 1636-1641.

11. Hiatt JR, Gabbay J, Busuttil RW (1994) Surgical anatomy of the hepatic arteries in 1000 cases. Ann Surg, 220: 50-52.

12. lezzi $R$, Cotroneo $A R$, Giancristofaro $D$, Santoro $M$, Storto $M L$ (2008) Multidetector-row CT angiographic imaging of the celiac trunk: anatomy and normal variants. Surg Radiol Anat, 30: 303-310.

13. Itri JN, Heller MT, Tublin ME (2013) Hepatic transplantation: postoperative complications. Abdom Imag, 38:1300-1333.

14. Lippert H, Pabst R (1985) Arterial variations in man. JF Bergmann, Munich, pp. 30-31.

15. Lipshutz B (1917) A composite study of the coeliac axis artery. Ann Surg, 65: 159-169.

16. Matusz P, Loukas M, lacob N, Ples H (2012) Common stem origin of left gastric, right and left inferior phrenic arteries, in association with a hepatosplenomesenteric trunk, independently arising from the abdominal aorta: case report using MDCT angiography. Clin Anat, 26:980-983.

17. McNulty JG, Hickey N, Khosa F, O Brien P, O Callaghan JP (2001) Surgical and radiological significance of variants of Bühler's anastomotic artery: a report of three cases. Surg Radiol Anat, 23: 277-280.

18. Morita M (1935) Reports and conception of three anomalous cases in the area of the celiac and the superior mesenteric arteries. Igaku Kenkyu, 9: 1993-2006.

19. Nakamura Y, Miyaki T, Hayashi S, limura A, Itoh M (2003) Three cases of the gastrosplenic and the hepatomesenteric trunks. Okajimas Folia Anat Jpn, 80: 71-76.

20. Nayak S. R., Latha V. Prabhu, A. Krishamurthy, C. Ganesh Kumar, Lakshmi A. Ramanathan, Abhijith Acharya, Abhishek Prasad Sinha (2008) Additional branches of celiac trunk and its clinical significance. Rom J Morphol Embryol, 49: 247-249.

21. Nelson TM, Polak R, Jonasson O, Abcarian H (1998). Anatomic variants of the celiac, superior mesenteric, and inferior mesenteric arteries and their clinical relevance. Clin Anat, 1: 75-91.

22. Oran I, Yesildag A, Memis A (2001) Aortic origin of right hepatic artery and superior mesenteric origin of splenic artery: two rare variations demonstrated angiographically. Surg Radiol Anat, 23: 349-352.

23. Prakash, Mokhasi V, Rajini T, Shashirekha M (2011) The abdominal aorta and its branches: anatomical variations and clinical implications. Folia Morphol, 70: 282.

24. Saad WE, Davies MG, Sahler L, Lee D, Patel N, Kitanosono T, Sasson T, Waldman D (2005) Arc of buhler: incidence and diameter in asymptomatic individuals. Vasc Endovascular Surg, 39: 347-349.

25. Sadler TW (1990) Langman's medical embryology. 6th Ed. William-Wilkins, Baltimore, pp. 198-202.

26. Sato M, Anno I, Yamaguchi M, lida H, Orii K (2006) Splenic artery aneurysm of the anomalous splenomesenteric trunk: successful treatment by transcatheterembolization using detachable coils. Cardiovasc Intervent Radiol, 29: 432-434.

27. Settembrini PG, Jausseran JM, Roveri S, Ferdani M, Carmo M, Rudondy P, Serra MG, Pezzuoli G (1996) Aneurysms of anomalous splenomesenteric trunk: clinical features and surgical anagement in two cases. J Vasc Surg, 24: 687-692.

28. Shukla PJ, Barreto SG, Kulkarni A, Nagarajan G, Fingerhut A (2010) Vascular anomalies encountered during pancreatoduodenectomy: do they influence outcomes. Ann Surg Oncol, 17: 186-193.

29. Skandalakis $\sqcup$, Rowe JS Jr, Gray SW, Skandalakis JE (1993) Surgical embryology and anatomy of the pancreas. Surg Clin North Am, 73: 661-697.

30. Song SY, Chung JW, Yin YH, Jae HJ, Kim HC, Jeon UB, Cho BH, So YH, Park JH (2010) Celiac axis and common hepatic artery variations in 5002 patients: systematic analysis with spiral CT and DSA. Radiology, 255: 278-288.

31. Stanley JC, Thompson NW, Fry WI (1970). Splanchnic artery aneurysms. Arch Surg, 101: 689-697.

32. Suma Y, Ramachandra rao K, Jayanthi V (2010) Splenomesenteric trunk - a case report. Anatomica Karnataka, 4: 12-14.

33. Tandler J (1904) U"ber die Varietaten der Arteria coeliaca and deren Entwicklung. Mat Hefte, 25: 473-500.

34. Uflacker R (1997) Atlas of vascular anatomy: an angiographic approach. Baltimore: Williams-Wilkins. p. 811.

35. Ugurel M S, Battal B, Bozlar U, Nural M S, Tasar M, Ors F, Saglam M, Karademir I (2010) Anatomical variations of hepatic arterial system, coeliac trunk and renal arteries: an analysis with multidetector CT angiography. Br J Radiol, 83: 661-667.

36. Vandamme JP, Bonte J (1985). The branches of the celiac trunk. Acta Anat (Basel), 122: 110-114.

37. Venieratos D, Panagouli E, Lolis E, Tsaraklis A, Skandalakis $P$ (2012) A morphometric study of the celiac trunk and review of the literature. Clin Anat, 26: 741-750.

38. Yíldírím M, Celik HH, Yíldíz Z, Tatar I, Aldur MM (2004) The middle colic artery originating from the coeliac trunk. Folia Morphol, 63: 363-365. 